\title{
Should Pulmonary Rehabilitation be a standard of care in lung cancer?
}

\section{Michael Steiner}

Institute for Lung Health

Leicester Biomedical Research Centre - Respiratory

University of Leicester

Glenfield Hospital

Groby Road

Leicester

LE3 9QP*

Email: $\quad \underline{m s 346 @ l e . a c . u k}$

* Address for Correspondence 
There are few therapeutic interventions as effective as Pulmonary Rehabilitation (PR) in the management of chronic respiratory disease. The scientific evidence base demonstrating the clinical benefit of PR is incontestable, indeed the most recent iteration of the Cochrane systematic review summarising clinical trials of PR in COPD concluded that no further clinical trials testing effectiveness were needed. ${ }^{1}$ The gains in physical capacity and health related quality of life conferred by PR in routine clinical practice are comparable to those seen in clinical trials ${ }^{2}$ and the available evidence suggests that completion of PR reduces subsequent healthcare costs through a reduction in the number of days spent in hospital. ${ }^{3}$ This substantial body of evidence is derived largely from studies in people with COPD but perhaps unsurprisingly, PR is also effective in other chronic respiratory diseases characterised by exercise limitation due to dyspnoea and muscle fatigue such as bronchiectasis, interstitial lung disease and pulmonary hypertension. This benefit, long understood by rehabilitation practitioners in the field, is increasingly now backed up by clinical trials of PR in these specific patient populations. ${ }^{4-6}$

In this issue of the journal, Edbrooke and colleagues further extend our understanding of the broader role of PR in respiratory disease by undertaking a clinical trial of a home based rehabilitation intervention in patients receiving non-surgical therapy for lung cancer. ${ }^{7}$ The trial aimed to encourage participation in an 8-week programme of structured, progressive physical activity and exercise whilst volunteers were also attending medical centres for active cancer treatment (chemo- or radiotherapy, commenced no more than 4 weeks prior to enrolment). On the face of it there is a strong rationale for such an intervention; patients presenting with inoperable lung cancer frequently have low performance status, poor exercise capacity and are physically inactive. ${ }^{8}$ Rehabilitation has the potential to improve quality of life by modifying these deficits and could theoretically increase the likelihood of successful completion of active cancer therapy. However, the trial did not show a statistically significant between group difference in 6 minute walking distance (6MWD) at 9 weeks (the primary endpoint) although there were significant differences in health status in favour of the rehabilitation group after 6 months. The authors considered adherence to the exercise component of the intervention to be sub-optimal with only around half the participants in the rehabilitation group maintaining adherence to the prescribed training component, speculating that this was the explanation for the lack of efficacy. 
The authors can be congratulated on successfully conducting a detailed and well-designed trial in a group of patients likely to have a significant rehabilitation need. The study was adequately assessor blinded and the sample size sufficient to detect the a priori defined meaningful treatment effect for the primary endpoint. The lack of efficacy (at least for the primary outcome measure) might also have been explained by the outcome measure chosen and the setting of the rehabilitation intervention. Patients were excluded if they reported not achieving guideline prescribed levels of physical activity in the previous month. However, given that the month in question must have been the immediate aftermath of a diagnosis of lung cancer, this estimate may not have been representative of the subjects normal activity (and thereby fitness). In support of this, values for 6MWD at baseline were not especially low and this might have reduced the sensitivity of the 6MWD to detect improvement in physical performance because of a ceiling effect. The authors chose to deliver a home based, rather than directly supervised rehabilitation intervention, presumably because this was felt to be more acceptable/practical for volunteers who were also attending healthcare centres regularly for cancer treatment. There are indications from recent trials in COPD that home or self managed PR may have equivalent efficacy to conventional supervised programmes, although the magnitude of benefit seen across treatment arms in these trials have not always matched those seen for conventional PR in clinical practice ${ }^{910}$. It is possible that the challenge of seeing through active chemo- or radiotherapy, combined with rather distant supervision of the programme (mostly by telephone) resulted in insufficient intensity of the exercise component to translate into a meaningful change in exercise performance.

The study adds to literature on rehabilitation/exercise interventions in patients with advanced non-resectable lung cancer summarised in a recent Cochrane systematic review. ${ }^{11}$ Whilst the authors of this review considered the quality of the evidence to be low and the risk of bias high, the meta-analyses performed did indicate that there were positive effects of exercise based rehabilitation programmes on walking performance and health related quality of life for those patients that complete them. It is also clear from scrutiny of the studies included in this review, that participants varied in their receipt of active non-surgical cancer therapy and that the timing and supervision of the rehabilitation intervention was similarly variable. 
These findings together with the study by Edbrooke et al present a complex evidence landscape framing the provision of rehabilitation in patients with advanced lung cancer in clinical practice. We can simply extrapolate the likely benefit of exercise training in terms of physical performance from trials in other respiratory populations (and indeed healthy, older people) but this would ignore the unique set of circumstances pertaining to patients who have had a recent diagnosis of lung cancer and are faced with the fearfulness of an uncertain prognosis and the prospect of undertaking chemotherapy and/or radiotherapy.

A diagnosis of cancer can be considered as a "biographically disruptive event", a term first described by Bury ${ }^{12}$ in relation to the onset of chronic disease as the disruption to an individual's expectation of life trajectory and personal biography. ${ }^{13}$ These circumstances might present a substantial barrier to active engagement in progressive exercise rehabilitation, when attention will be focussed on coming to terms with the diagnosis and prospect of undertaking cancer treatment and readiness to engage in PR might be low. In this respect there is a contrast to the situation in COPD, where the indolent nature of the condition without a clear initiating event has been identified as an indicator of a lack of biographical disruption and a more passive acceptance of the constraints of the disease. ${ }^{14}$ How these different disease narratives impact readiness to undertake pulmonary rehabilitation, which can only be effective with the active engagement of the recipient, is unknown. In an insightful investigation of the views of patients living with advanced lung cancer about exercise therapy, Cheville et al reported that "highly structured, non-daily activity-oriented exercise programs will be unappealing and poorly accepted by this population without careful planning and implementation". ${ }^{15}$ Some patients however, were "robustly, and perhaps defiantly, active" and a common theme was the view that specific exercise recommendations made by their oncologist would carry substantial weight. The timing and source of a rehabilitation offer for lung cancer sufferers will clearly be crucial. In the absence of more robust evidence about the optimal timing of the offer of PR after a diagnosis of lung cancer, referrers and practitioners will need to take a judgement in individual cases whether the intervention might be more effectively initiated after active cancer treatment has been completed because this might be the moment when the patient will be most open to behaviour change with the objective of improving physical activity and 
health and enhancing survival chances. Interestingly and in support of this, in the study by Edbrooke et al, there was a greater increment in 6MWD between the 9 week and 6 month timepoints in the rehabilitation group; the latter presumably after active cancer treatment had been completed. Indeed, the between group difference in 6MWD at 6 months (in the modified intention to treat analysis) was $41 \mathrm{~m}$ which is higher than the recently revised MCID but lower than the difference the study was powered to detect. This, married to the gains in health status observed at 6 months, suggests the intervention might have brought about an important albeit delayed health benefit.

Perhaps the most tantalising finding of the study by Edrooke et al was the observation of better survival in the active rehabilitation group. The authors rightly do not place too much emphasis on this finding as the study was not powered to investigate mortality and the observed difference was not statistically significant. However, the finding is plausible and in line with epidemiological evidence that higher physical activity and fitness is associated with better long term cancer survival. ${ }^{16}$ This together with a robust trial evidence that exercise rehabilitation in other cancer types is effective in improving symptoms and quality of life has prompted the Clinical Oncology Society of Australia to publish a position statement recommending exercise is prescribed to all cancer sufferers as part of their treatment regimen. ${ }^{17}$ As developments in targeted biologic therapy advance, the impact and prognosis of lung cancer may improve such that the time-course of the condition could become more akin to a chronic disease. Whilst there is much to learn about how it should be integrated with active cancer therapy, there is little doubt that exercise based therapies such as Pulmonary Rehabilitation will have an important role to play. 


\section{References}

1. Steiner M, McMillan V, Lowe D, et al. Pulmonary rehabilitation: An exercise in improvement. National Chronic Obstructive Pulmonary Disease (COPD) Audit Programme: Clinical and organisational audits of pulmonary rehabilitation services in England and Wales 2017. National report. : Royal College of Physicians, 2018.

2. Steiner M, Holzhauer-Barrie J, Lowe D, et al. Pulmonary Rehabilitation: Steps to breathe better. National Chronic Obstructive Pulmonary Disease (COPD) Audit Programme: Clinical Audit of Pulmonary Rehabilitation services in England and Wales 2015. London: Royal College of Physicians, 2016.

3. Steiner M MV, Lowe D, Saleem Khan M, Holzhauer-Barrie J, Van Loo V, Roberts CM. Pulmonary rehabilitation: Beyond breathing better. National Chronic Obstructive Pulmonary Disease (COPD) Audit Programme: Outcomes from the clinical audit of pulmonary rehabilitation services in England 2015. London: Royal College of Physicians, 2017.

4. Morris NR, Kermeen FD, Holland AE. Exercise-based rehabilitation programmes for pulmonary hypertension. Cochrane Database Syst Rev 2017;1:CD011285. doi: 10.1002/14651858.CD011285.pub2

5. Lee AL, Hill CJ, Cecins N, et al. The short and long term effects of exercise training in non-cystic fibrosis bronchiectasis--a randomised controlled trial. Respir Res 2014;15:44. doi: 10.1186/1465-9921-15-44

6. Dowman L, Hill CJ, Holland AE. Pulmonary rehabilitation for interstitial lung disease. Cochrane Database Syst Rev 2014(10):CD006322. doi: 10.1002/14651858.CD006322.pub3

7. Edbrooke L, Aranda S, Granger CL, et al. Multidisciplinary home-based rehabilitation in inoperable lung cancer: a randomised controlled trial. Thorax 2019; In Press

8. Granger $\mathrm{CL}, \mathrm{McD}$ onald $\mathrm{CF}$, Irving $\mathrm{L}$, et al. Low physical activity levels and functional decline in individuals with lung cancer. Lung Cancer 2014;83(2):292-9. doi: 10.1016/j.lungcan.2013.11.014

9. Holland AE, Mahal A, Hill CJ, et al. Home-based rehabilitation for COPD using minimal resources: a randomised, controlled equivalence trial. Thorax 2017;72(1):57-65. doi: 10.1136/thoraxjnl-2016-208514

10. Horton EJ, Mitchell KE, Johnson-Warrington V, et al. Comparison of a structured home-based rehabilitation programme with conventional supervised pulmonary rehabilitation: a randomised non-inferiority trial. Thorax 2018;73(1):29-36. doi: 10.1136/thoraxjnl-2016-208506 
11. Peddle-McIntyre CJ, Singh F, Thomas R, et al. Exercise training for advanced lung cancer. Cochrane Database Syst Rev 2019;2:CD012685. doi:

10.1002/14651858.CD012685.pub2

12. Bury M. Chronic illness as biographical disruption. Sociol Health IIIn 1982;4(2):16782.

13. Hubbard G, Forbat L. Cancer as biographical disruption: constructions of living with cancer. Support Care Cancer 2012;20(9):2033-40. doi: 10.1007/s00520-011-1311-9

14. Pinnock $H$, Kendall $M$, Murray SA, et al. Living and dying with severe chronic obstructive pulmonary disease: multi-perspective longitudinal qualitative study. $B M J$ 2011;342:d142. doi: 10.1136/bmj.d142

15. Cheville AL, Dose AM, Basford JR, et al. Insights into the reluctance of patients with late-stage cancer to adopt exercise as a means to reduce their symptoms and improve their function. J Pain Symptom Manage 2012;44(1):84-94. doi: 10.1016/j.jpainsymman.2011.08.009

16. Cormie P, Zopf EM, Zhang X, et al. The Impact of Exercise on Cancer Mortality, Recurrence, and Treatment-Related Adverse Effects. Epidemiol Rev 2017;39(1):7192. doi: 10.1093/epirev/mxx007

17. Australia COSo. COSA position statement on exercise in cancer care, 2018. https://www.cosa.org.au/media/332488/cosaposition-statement-v4-webfinal.pdf 